Pesq. Vet. Bras. 30(7):541-546, julho 2010

\title{
Doença tremorgênica em ruminantes e equídeos no semiárido da Paraíba ${ }^{1}$
}

\author{
Clarice R.M. Pessoa ${ }^{2}$, Rosane M.T. Medeiros ${ }^{2}$, André F.A. Pessoa², Antônio \\ F.M. Dantas ${ }^{2}$, Odaci F. de Oliveira ${ }^{3}$ e Franklin Riet-Correa ${ }^{2}$
}

\begin{abstract}
Pessoa C.R.M., Medeiros R.M.T., Dantas A.F., Oliveira O.F. \& Riet-Correa F. 2010. [Tremorgenic disease in ruminants and equidae in the Brazilian semiarid.] Doença tremorgênica em ruminantes e equídeos no semiárido da Paraiba. Pesquisa Veterinária Brasileira 30(7):541-546. Hospital Veterinário, Centro de Saúde e Tecnologia Rural, Universidade Federal de Campina Grande, Campus de Patos, 58700-970 Patos, PB, Brazil. E-mail: rmtmed@uol.com

Eight outbreaks of a tremorgenic disease are reported in ruminants and equidae in the semiarid region of the Brazilian state of Paraíba. Seven outbreaks occurred from July to December 2007, with highest frequency in September and October. Another outbreak was observed in February 2008. All outbreaks occurred during the dry season. The disease affected horses, mules, cattle and sheep. Clinical signs were staggering, hypermetria, ataxia, widebased stance and alertness. After being removed from pastures the animals recovered in a period of 3-4 days to two weeks, however when returned to the pasture clinical signs reappeared. One affected sheep was euthanized and necropsied. Gross and histologic lesions were not observed. Different grasses including Digitaria bicornis, Enteropogon mollis, Chloris virgata and Chloris barbata were present in the pastures where the disease occurred. In six farms the disease occurred in cultures of Opuntia ficcus-indica invaded by grasses and two in deforested native vegetation invaded by similar grasses. Two horses were fed ad libitum during seven days, mature forage collected in pastures where the disease occurred. One horse showed mild signs of the disease on the fifth day of consumption, but recovered one day later. These results suggest that the disease is associated with the ingestion of some graminae, probably Chloris spp. Previous reports mentioned the occurrence of a similar disease, between 1956 and 1962, in the semiarid region of Pernambuco, in pastures with Chloris orthonothon.
\end{abstract}

INDEX TERMS: Poisonous plants, tremorgenic toxins, plant poisoning, cattle, horses, mules, sheep, semiarid.

RESUMO.- Descrevem-se oito surtos de uma doença tremorgênica em bovinos, ovinos, equinos e muares na região do Cariri, semiárido da Paraíba. Sete surtos aconteceram de julho a dezembro de 2007, com maior frequência entre setembro e outubro. Outro surto foi observado em fevereiro de 2008. Todos os surtos ocorreram no período da seca. Os sinais observados foram tremores musculares, hipermetria, ataxia, aumento da base de sustentação, cons-

\footnotetext{
${ }^{1}$ Recebido em 28 de dezembro de 2009.

Aceito para publicação em 14 de janeiro de 2010.

${ }^{2}$ Hospital Veterinário, Centro de Saúde e Tecnologia Rural (CSTR), Universidade Federal de Campina Grande, Campus de Patos, 58700970 Patos, PB, Brasil. *Autor para correspondência: rmtmed@uol.com

${ }^{3}$ Veterinário Autônomo, Cx. Postal 117, Mossoró, RN 59600-970, Brasil.
}

tante estado de alerta e, em alguns casos, decúbito. Quando retirados das pastagens os animais recuperavam-se em 3-4 dias a duas semanas, porém quando retornavam ao pasto de origem adoeciam novamente. Um ovino foi necropsiado e não foram observadas lesões macroscópicas ou microscópicas. Em seis propriedades a doença ocorreu em cultivos de palma invadidos por gramíneas e em duas em áreas de caatinga desmatada invadidas pelas mesmas gramíneas. Diversas gramíneas, incluindo Digitaria bicornis, Enteropogon mollis, Chloris virgata e Chloris barbata foram encontradas nos piquetes onde ocorreu a doença. Dois equinos foram alimentados por sete dias com gramíneas secas provenientes de fazendas onde haviam acontecido surtos da doença. Um dos equinos apresentou sinais leves da doença no quinto dia de ingestão, mas recuperou-se no dia seguinte. 
Esses resultados sugerem que a doença está associada à ingestão de alguma gramínea, possivelmente Chloris spp. Relatos anteriores mencionam a ocorrência de uma intoxicação semelhante, entre 1956 e 1962, no Agreste Pernambucano, em pastagens de Chloris orthonothon.

TERMOS DE INDEXAÇÃO: Plantas tóxicas, toxinas tremorgênicas, intoxicação por planta, bovinos, equinos, muares, ovinos, semiárido.

\section{INTRODUÇÃO}

Doenças tremorgênicas são toxicoses caracterizadas por tremores musculares, ataxia, hipermetria, aumento da base de sustentação, desequilíbrio, atitude de alerta, decúbito e, em raros casos, morte. Geralmente os sintomas se exacerbam pela estimulação externa ou quando os animais são movimentados. Na maioria das vezes, os animais, quando são retirados das pastagens, recuperam-se espontaneamente (Radostits et al. 2007). São descritos como agentes causadores da síndrome diversas gramíneas ou fungos e bactérias que as infectam, incluindo Poa huecu (Carrillo et al. 1983), Phalaris spp., Cynodon dactylon, Paspalum spp. infectado por Claviceps paspali, Lolium perenne infectado por Neotyphodium Iolii e Lolium rigidum infectado pela bactéria Clavibacter toxicus (Radostitis et al. 2007). No Brasil são relatadas doenças tremorgênicas causadas por Phalaris angusta (Gava et al. 1999), Ipomoea asarifolia (Tokarnia et al. 2000, Medeiros et al. 2003, Araújo et al. 2008, Tortelli et al. 2008) e Paspalum spp. infectado por $C$. paspali (Riet-Correa et al. 1983). Na maioria dos casos não há lesões patológicas relacionadas, exceto na intoxicação por Phalaris spp., onde a lesão patológica característica é pigmentação cinza-esverdeada no sistema nervoso central (Gava et al. 1999). Em casos de intoxicação por Lolium perenne (Mason 1968), Ipomoea asarifolia (Guedes et al. 2007) e Clavipes paspali (Riet-Correa et al. 1993) descreve-se degeneração de células de Purkinje associada a esferóides axonais na camada granular do cerebelo. Outros fungos como Penicillium cyclopium e Aspergillus clavatus, que infectam grãos e resíduos de cervejaria, podem produzir toxinas que ocasionam a doença (RietAlvariza 1993).

No Nordeste a única causa de doença tremorgênica é Ipomoea asarifolia (Medeiros et al. 2003, Araújo et al. 2008), porém existem relatos da doença ainda não esclarecidos quanto ao agente causador. Um deles descreve a existência do "mal-do-tombo" no Agreste de Pernambuco, atingindo bovinos entre 1956 e 1962 (Anônimo 1962, Tokarnia 1962). Um dos autores (Riet-Correa, dados não publicados) observou, em 2005, essa síndrome em vacas leiteiras e equinos em regime de pastejo no município de São José do Campestre, Rio Grande do Norte.

Este trabalho tem como objetivo descrever surtos de uma síndrome tremorgênica em equídeos e ruminantes ocorridos entre 2007 e 2008 em alguns municípios da região semiárida da Paraíba. Para isso sinais clínicos e dados epidemiológicos foram coletados em visitas a propriedades onde aconteceram os surtos. Adicionalmente foram desenvolvidos experimentos na tentativa de reproduzir a doença.

\section{MATERIAL E MÉTODOS}

Os dados epidemiológicos e os sinais clínicos foram coletados durante a ocorrência dos surtos em oito fazendas onde ocorreu a doença, sete em 2007 e uma em 2008. Em 2008, depois do início das chuvas, novas visitas foram feitas a todas as propriedades para coleta das gramíneas dominantes em cada pastagem para identificação botânica.

Um ovino procedente do município de Soledade foi eutanasiado e amostras de órgãos da cavidade abdominal, torácica e sistema nervoso central (SNC) foram coletados e fixadas em formol tamponado a $10 \%$, incluídos em parafina, processados rotineiramente e corados por hematoxilina-eosina para estudos histológicos. O estudo histológico do SNC foi realizado em cortes transversais do córtex frontal, parietal, temporal e occipital, núcleos da base, tálamo, colículos rostral e caudal, ponte e pedúnculos cerebelares, bulbo na altura do óbex, cerebelo, medulas cervical, torácica e lombar, gânglio e nervo trigêmeo.

$\mathrm{Na}$ tentativa de reproduzir experimentalmente a doença, dois equinos (um jovem e um adulto), foram mantidos em baias individuais por sete dias e alimentados com gramíneas secas ad libitum, provenientes de uma fazenda onde havia acontecido um surto da doença. A forragem era coletada a cada dois dias e acondicionada em sacos de náilon. Para determinação da presença de fungos endofíticos nas gramíneas, amostras foram enviadas ao Dr. Ernesto Odriozola do Instituto Nacional de Tecnologia Agrícola (INTA) em Balcarce, Argentina.

\section{RESULTADOS}

\section{Dados epidemiológicos e sinais clínicos}

A doença atingiu equinos, muares, bovinos e ovinos que se alimentavam de diferentes tipos de pastagem (Quadro 1), na época de seca entre 2007 e 2008. Os sinais clínicos apresentados foram tremores musculares, aumento da base de sustentação, ataxia, hipermetria, constante estado de alerta e quedas (Fig.1). Esses sinais se exacerbavam quando os animais eram manejados. Em alguns casos mais graves ocorria decúbito permanente. $\mathrm{O}$ apetite era mantido. As taxas de morbidade e mortalidade estão no Quadro 1. Dois cavalos que morreram estavam muito debilitados em consequência da seca. Quando retirados da pastagem os animais recuperavam-se dentro de um período variável, de 3-4 dias a duas semanas, e a recuperação era completa. Alguns animais que foram retornados ao pasto de origem adoeceram novamente. Em um dos casos uma égua com cria ao pé adoeceu, porém o potro não apresentou qualquer sinal clínico da doença.

A doença ocorreu somente em alguns piquetes de cada propriedade, atingindo animais nativos e recém introduzidos, sendo que nesses últimos o quadro clínico iniciavase cerca de 1-2 dias depois da introdução. Na Fazenda 2 foram afetados dois equinos e um muar, que ao serem retirados do piquete se recuperaram em 3-7 dias. Um dos 
Quadro 1. Características epidemiológicas da síndrome tremorgênica observada no semiárido paraibano em diferentes espécies animais

\begin{tabular}{|c|c|c|c|c|c|c|c|}
\hline Fazenda & $\begin{array}{l}\text { Espécies e nú- } \\
\text { mero de animais }\end{array}$ & $\begin{array}{l}\text { Morbidade } \\
\text { N (\%) }\end{array}$ & $\begin{array}{l}\text { Mortalidade } \\
\text { N (\%) }\end{array}$ & Época do anoa & $\begin{array}{c}\text { Espécies } \\
\text { dominantes }\end{array}$ & $\begin{array}{c}\text { Tipo de } \\
\text { pastagem }\end{array}$ & Município \\
\hline 1 & 4 equinos & $3(75 \%)$ & 0 & $\begin{array}{l}\text { Julho, Agosto } \\
\text { e Setembro }\end{array}$ & $\begin{array}{l}\text { Enteropogon mollis } \\
\text { e Chloris spp. }\end{array}$ & $\begin{array}{l}\text { Cultura antiga } \\
\text { de palma }\end{array}$ & Cabaceiras \\
\hline 2 & $\begin{array}{l}2 \text { equinos } \\
2 \text { muares }\end{array}$ & $\begin{array}{c}2(100 \%) \\
1(50 \%)\end{array}$ & 0 & $\begin{array}{l}\text { Setembro, Outubro } \\
\text { e Novembro }\end{array}$ & Chloris spp. & Cultivo de palma & Campina Grande \\
\hline 3 & 7 equinos & $3(43 \%)$ & $2(28,5 \%)$ & Outubro e Dezembro & $\begin{array}{l}\text { Enteropogon mollis } \\
\text { e Chloris spp. }\end{array}$ & Cultivo de palma & Boa Vista \\
\hline 4 & 1 equino & $1100 \%$ & 0 & Dezembro & $\begin{array}{l}\text { Enteropogon mollis } \\
\text { e Chloris spp. }\end{array}$ & Cultivo de palma & Boa Vista \\
\hline 5 & $\begin{array}{l}40 \text { bovinos } \\
7 \text { equinos }\end{array}$ & $\begin{array}{l}3(7,5 \%) \\
3(43 \%)\end{array}$ & 0 & Setembro e Outubro & $\begin{array}{l}\text { Enteropogon mollis } \\
\text { e Chloris spp. }\end{array}$ & Caatinga desmatada & Boa Vista \\
\hline 6 & 7 equinos & $4(57 \%)$ & 0 & $\begin{array}{c}\text { Setembro, Outubro } \\
\text { e Novembro }\end{array}$ & $\begin{array}{l}\text { Enteropogon mollis } \\
\text { e Chloris spp. }\end{array}$ & Cultivo de palma & Boa Vista \\
\hline 7 & 72 ovinos & $19(26 \%)$ & $4(5,5 \%)$ & Setembro & $\begin{array}{l}\text { Enteropogon mollis } \\
\text { e Chloris spp }\end{array}$ & Caatinga desmatada & Soledade \\
\hline 8 & 1 equino & $1(100 \%)$ & 0 & Fevereiro & $\begin{array}{l}\text { Enteropogon mollis } \\
\text { e Chloris spp. }\end{array}$ & Cultivo de palma & Boa Vista \\
\hline
\end{tabular}

\footnotetext{
a Os Surtos 1-7 ocorreram em 2007 e o Surto 8 em 2008.
}
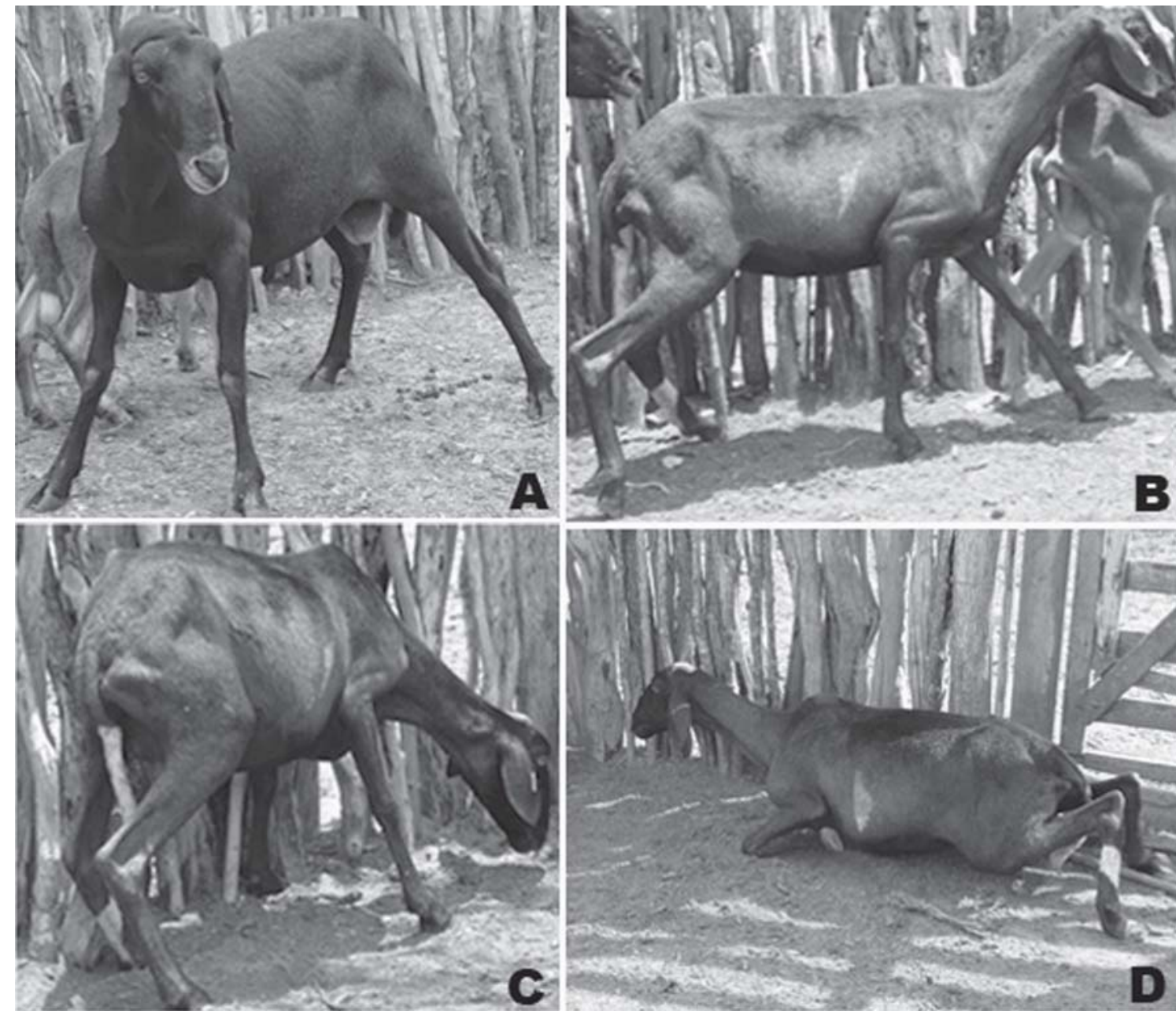

Fig.1. Ovinos com sinais tremorgênicos, (A) aumento da base de sustentação; (B) hipermetria; (C) perda de equilíbrio com tremores generalizados; (D) queda em posição não-usual. 
equinos, dois meses após a recuperação, foi reintroduzido na pastagem; 36 horas após mostrou sinais clínicos intensos que evoluíram rapidamente para o decúbito em 48 horas. Após ser retirado da pastagem tornou a se recuperar.

Alguns dados epidemiológicos dos surtos são apresentados no Quadro 1. Durante as visitas foi observado que a maioria dos piquetes nos quais os animais estavam eram de campos de palma forrageira (Opuntia fícus-indica). No período em que ocorreu a doença a região atravessava uma época de estiagem, a forragem de todos os piquetes onde ocorreram os surtos era constituída principalmente por talos de gramíneas, que se apresentavam secos, sem inflorescências, impedindo sua identificação. Em algumas fazendas encontravam-se diferentes plantas invasoras (Herssantia crispa, Jacquemontia sp. e Passiflora foetida). Alguns animais que estavam estabulados apresentaram sinais clínicos após receberem pasto coletado em piquetes com palma forrageira. As gramíneas coletadas no ano de 2008 nos locais onde ocorreram os surtos foram identificadas como Digitaria bicornis, Enteropogon mollis, Chloris virgata e Chloris barbata (Fig.2).
Não foram observados fungos endofíticos nas amostras enviadas ao Instituto Nacional de Tecnologia Agrícola (INTA). Em 2008 e 2009 a doença não tornou a ocorrer em nenhuma das propriedades nas quais tinha sido constatada no ano anterior, apesar dos produtores não terem introduzido mudanças importantes no manejo das pastagens. Nenhuma alteração macroscópica ou microscópica foi encontrada no ovino necropsiado.

\section{Reprodução experimental}

Um dos equinos alimentados com gramíneas coletadas na Fazenda 4 apresentou leves tremores nos músculos dos membros anteriores no quinto dia da administração da forragem seca. Esses sinais desapareceram no dia seguinte, apesar de a administração ter continuado ainda por mais dois dias. O outro animal não apresentou sinais após sete dias de consumo.

\section{DISCUSSÃO E CONCLUSÕES}

A observação dos dados epidemiológicos, clínicos e experimentais sugere que a doença descrita neste trabalho é causada por uma planta tóxica presente nas pastagens ou

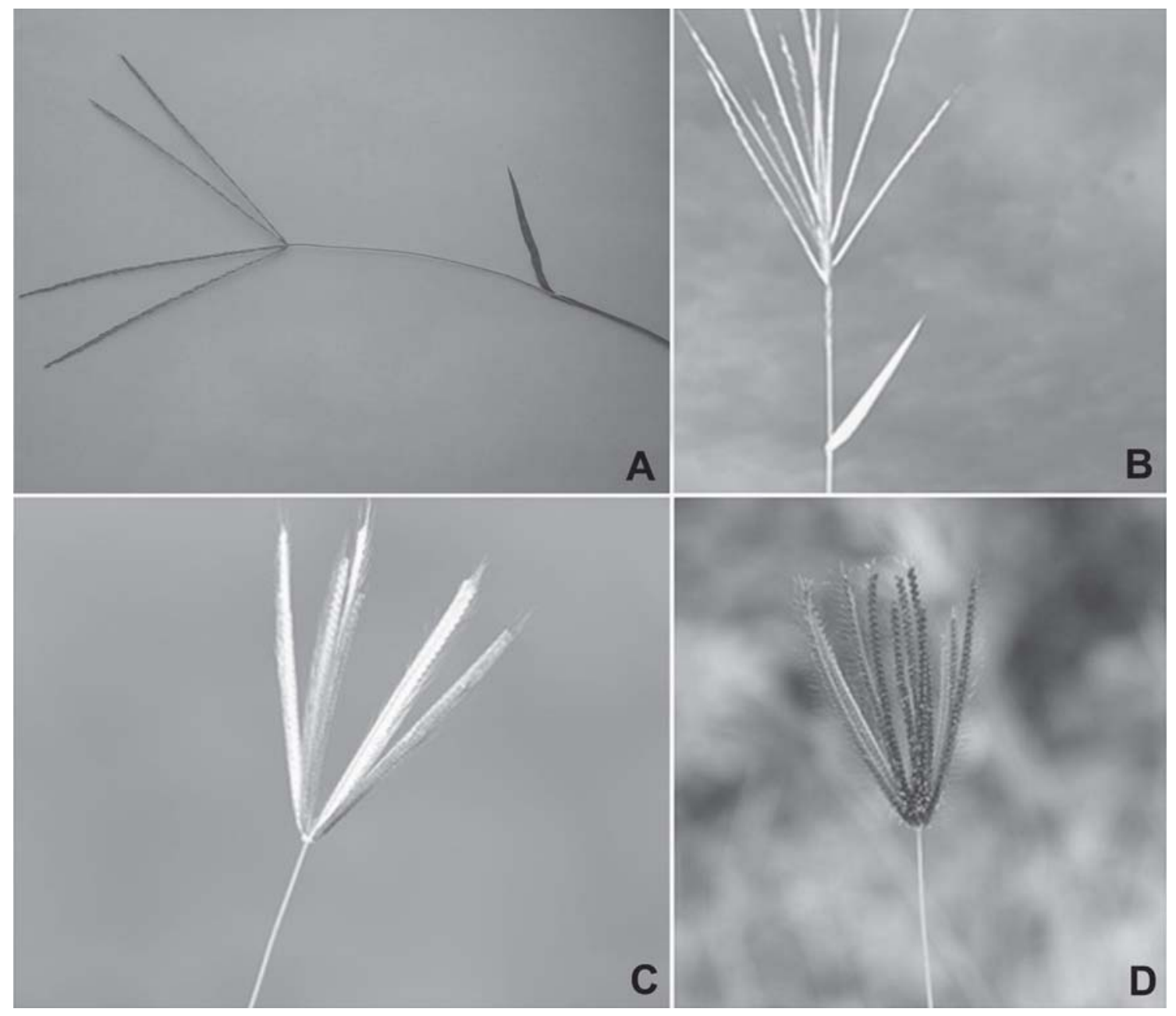

Fig.2. Gramíneas presentes em maior quantidade nas pastagens onde ocorreram surtos de síndrome tremorgênica. (A) Digitaria bicornis; (B) Enteropogon mollis; (C) Chloris virgata; (D) Chloris barbata. 
por uma toxina associada a essas plantas. As manifestações clínicas observadas nos animais afetados foram semelhantes aos causados por toxinas tremorgênicas mencionadas na introdução, incluindo as intoxicações por Paspalum spp. infectado por Claviceps paspali, Phalaris spp., Lolium multiflorum, Lolium perenne e Poa huecu, que não ocorrem na região. Outras plantas tremorgênicas encontradas na região são Cynodon dactylon e Ipomoea asarifolia, que não foram encontradas nos piquetes onde ocorreram os surtos.

Uma diferença entre as intoxicações por plantas mencionadas anteriormente e os surtos descritos neste trabaIho é a ocorrência da doença em equídeos, o que é raramente mencionado nas intoxicações por essas plantas tremorgênicas. No nordeste a intoxicação por Ipomoea asarifolia, que é frequente em ruminantes, nunca foi descrita em equinos.

No estudo epidemiológico a principal suspeita foi de que a intoxicação fosse causada por gramíneas que estavam secas no momento das visitas. A identificação dessas plantas, coletadas no ano de 2008 , mostraram que as mais frequentes são Chloris spp., que foram encontradas em quantidades significativas em todas as fazendas. Enteropogon mollis também foi muito frequente, sendo encontrada em quantidades significativas em sete das oito fazendas (Quadro 1). Esses resultados sugerem que a doença está associada à ingestão de alguma gramínea, possivelmente Chloris spp. Além disso, a administração experimental dessas espécies a dois cavalos induziu sinais discretos em um deles.

Algumas plantas invasoras que se encontravam ainda verdes durante as visitas (Herssantia crispa, Jacquemontia sp. e Passiflora foetida) surgiram como suspeitas; no entanto essas plantas não foram encontradas em todas as fazendas onde ocorreu a doença. Herssantia crispa e Jacquemontia sp. foram administradas experimentalmente a ovinos, caprinos ou equinos por um dos autores (C. Pessoa, dados não publicados) com resultados negativos. Passiflora foetida é uma planta cianogênica, com conteúdo variável de glicosídeos cianogênicos, que causa sinais clínicos diferentes aos observados nestes surtos (CarvaIho 2009).

Uma doença muito similar á descrita neste trabalho, conhecida como "mal do tombo", ocorreu no Agreste de Pernambuco, em bovinos, entre 1956 e 1962, na mesma época do ano, nos meses de setembro e outubro, desaparecendo em dezembro (Anônimo 1962, Tokarnia 1962). Ainda segundo os mesmos autores, a doença foi provavelmente causada por vegetal ou por fungos que o parasitam, e uma gramínea conhecida como capim-de-raiz (Chloris orthonothon Doell.) era encontrada em todos os piquetes onde ocorria a enfermidade. No caso de alguma gramínea vir a ser responsável pelo quadro clínico, é provável que o mesmo seja causado por um fungo endofítico, à semelhança do que ocorre com Lolium perenne infectado por Neotyphodium lolii (Radostits et al. 2007). No entanto, amostras enviadas para identificar esses fungos deram resultados negativos, porém deve-se considerar que as amostras enviadas estavam muito secas. Deve ser considerada, também, a possibilidade de a toxina responsável pelo quadro clínico ser produzida pela própria planta, como é o caso de Phalaris spp. e provavelmente Cynodon dactylon, cuja toxina não tem sido identificada. Esta última é tóxica quando está seca, principalmente depois de geadas (Riet-Correa 1993).

Os sinais clínicos descritos nos surtos ocorridos no Agreste Pernambucano (Tokarnia 1962), caracterizados por andar desequilibrado, quedas e constante estado de alerta, são semelhantes aos observados neste estudo, no entanto no Agreste Pernambucano apenas bovinos foram atingidos, enquanto que nos casos ocorridos na Paraíba a doença atingiu bovinos, ovinos, equinos e muares. Um fato interessante é que todos os equinos afetados estavam em cultivos de palma, onde não havia outras espécies animais, ou eram alimentados com gramíneas coletadas nesses cultivos.

Em outro surto de doença tremorgênica em bovinos, no Rio Grande do Norte, foram afetados bovinos e equinos, mas na ficha consultada não foi mencionada a presença de Chloris spp. ou outra gramínea suspeita (Riet-Correa, dados não publicados).

Um fato importante é que a doença aparentemente não ocorre todos os anos, no entanto, alguns produtores mencionaram que a mesma fora observada em anos anteriores, mas com pequena incidência. A determinação da causa da doença e o estudo da epidemiologia da mesma são necessários para a adoção de medidas de controle e profilaxia. Até o momento a única recomendação a ser dada aos produtores é a retirada imediata dos animais afetados das pastagens, evitando movimentá-los até a completa recuperação.

\section{REFERÊNCIAS}

Anônimo. 1962. Tombo acabou de repente no agreste de Pernambuco. Correio da Manhã de 11.2.62., Recife, p.9.

Araújo J.A.S., Riet-Correa F., Medeiros R.M.T., Soares M.P., Oliveira D.M. \& Carvalho F.K.L. 2008. Intoxicação experimental por Ipomoea asarifolia (Convolvulaceae) em ovinos e caprinos. Pesq.Vet. Bras. 28(10):488-494

Carrillo B.J., Corbellini C.N. \& Viera B.F.J. 1983. Efecto tremorgénico y estudio patológico de la intoxicación experimental por Poa huecu. Revta Med. Vet., Buenos Aires, 64:152-164.

Carvalho F.K.L. 2009. Intoxicação experimental por Passiflora sp. em caprinos. Monografia de Graduação em Medicina Veterinária, Centro de Saúde e Tecnologia Rural, Universidade Federal de Campina Grande, Patos, PB. 29p.

Gava A., Sousa R.S, de Deus M.S., Pilati C., Cristani J., Mori A.M. \& Neves D.S. 1999. Phalaris angusta (Gramineae) como causa de enfermidade neurológica em bovinos no Estado de Santa Catarina. Pesq. Vet. Bras. 19(1):35-38.

Guedes K.M.R., Riet-Correa F., Dantas A.F.M., Simões S.V.D., Miranda Neto E.G., Nobre V.M.T. \& Medeiros R.M.T. 2007. Doenças do sistema nervoso central em caprinos e ovinos no semi-árido. Pesq. Vet. Bras. 27 (1):29-30.

Mason R.W. 1968. Axis cylinder degeneration associated with ryegrass staggers in sheep and cattle. Aust. Vet. J. 44:428. 
Medeiros R.M.T., Barbosa R.C., Riet-Correa F., Lima E.F., Tabosa I.M., Barros S.S. Gardner D.R. \& Molyneux R.J. 2003. Tremorgenic syndrome in goats caused by Ipomoea asarifolia in Northeastern Brazil. Toxicon 41:933-935.

Radostits O.M., Gay C.C., Blood D.C., Hinchcliff K.W. \& Constable P.D. 2007. Veterinary Medicine. $10^{\text {th }}$ ed. W.B. Saunders, London, p.1889-1912.

Riet-Alvariza F. 1993. Intoxicação por toxinas de Aspergillus spp. e Penicillium spp., p.137-141. In: Riet-Correa F., Méndez M.C. \& Schild A.L. (Eds), Intoxicação por Plantas e Micotoxicoses em Animais Domésticos. Editorial Agropecuária Hemisferio Sur, Montivideo.

Riet-Correa F. 1993. Intoxicação por Cynodon dactylon, p.127-131. In: Riet-Correa F., Méndez M.C. \& Schild A.L. (Eds), Intoxicação por
Plantas e Micotoxicoses em Animais Domésticos. Editorial Agropecuária Hemisferio Sur, Montivideo.

Riet-Correa F., Schild A.L., Méndez M.C., Tavares A.S. \& Rodrigues J.O. 1983. Intoxicação por Claviceps paspali em bovinos no Rio Grande do Sul. Pesq. Vet. Bras. 3(2):59-65.

Tokarnia C.H. 1962. Relatório de viagem à região do agreste do Estado de Pernambuco. Seção de Anatomia Patológica do Instituto de Biologia Animal, Rio de Janeiro, 9.fev.1962. (Mimeografado), 5p.

Tokarnia C.H., Döbereiner J. \& Peixoto P.V. 2000. Plantas que causam perturbações nervosas, p.123-125. In: Ibid. (Eds), Plantas Tóxicas do Brasil. Editora Helianthus, Rio de Janeiro.

Tortelli F.P., Barbosa J.D., Oliveira C.A., Riet-Correa F. \& Riet-Correa G. 2008. Intoxicação por Ipomoea asarifolia em ovinos e bovinos na Ilha de Marajó. Pesq. Vet. Bras. 28(12):622-626. 